\title{
Nonlocal Transport Processes and the Fractional Cattaneo-Vernotte Equation
}

\author{
J. F. Gómez Aguilar, ${ }^{1}$ T. Córdova-Fraga, ${ }^{2}$ J. Tórres-Jiménez, ${ }^{3}$ R. F. Escobar-Jiménez, ${ }^{4}$ \\ V. H. Olivares-Peregrino, ${ }^{4}$ and G. V. Guerrero-Ramírez ${ }^{4}$ \\ ${ }^{1}$ CONACYT-Centro Nacional de Investigación y Desarrollo Tecnológico, Tecnológico Nacional de México, \\ Interior Internado Palmira S/N, Colonia Palmira, 62490 Cuernavaca, MOR, Mexico \\ ${ }^{2}$ Departamento de Ingeniería Física, División de Ciencias e Ingenierías Campus León, Universidad de Guanajuato, \\ 37150 León, GTO, Mexico \\ ${ }^{3}$ Departamento de Electromecánica, Instituto Tecnológico Superior de Irapuato, 36821 Irapuato, GTO, Mexico \\ ${ }^{4}$ Centro Nacional de Investigación y Desarrollo Tecnológico, Tecnológico Nacional de México, Interior Internado Palmira S/N, \\ Colonia Palmira, 62490 Cuernavaca, MOR, Mexico
}

Correspondence should be addressed to J. F. Gómez Aguilar; jgomez@cenidet.edu.mx

Received 19 February 2016; Accepted 17 April 2016

Academic Editor: Juan J. Trujillo

Copyright (C) 2016 J. F. Gómez Aguilar et al. This is an open access article distributed under the Creative Commons Attribution License, which permits unrestricted use, distribution, and reproduction in any medium, provided the original work is properly cited.

\begin{abstract}
The Cattaneo-Vernotte equation is a generalization of the heat and particle diffusion equations; this mathematical model combines waves and diffusion with a finite velocity of propagation. In disordered systems the diffusion can be anomalous. In these kinds of systems, the mean-square displacement is proportional to a fractional power of time not equal to one. The anomalous diffusion concept is naturally obtained from diffusion equations using the fractional calculus approach. In this paper we present an alternative representation of the Cattaneo-Vernotte equation using the fractional calculus approach; the spatial-time derivatives of fractional order are approximated using the Caputo-type derivative in the range $(0,2]$. In this alternative representation we introduce the appropriate fractional dimensional parameters which characterize consistently the existence of the fractional space-time derivatives into the fractional Cattaneo-Vernotte equation. Finally, consider the Dirichlet conditions, the Fourier method was used to find the full solution of the fractional Cattaneo-Vernotte equation in analytic way, and Caputo and Riesz fractional derivatives are considered. The advantage of our representation appears according to the comparison between our model and models presented in the literature, which are not acceptable physically due to the dimensional incompatibility of the solutions. The classical cases are recovered when the fractional derivative exponents are equal to 1.
\end{abstract}

\section{Introduction}

Fourier's law satisfies the heat conduction induced by a small temperature gradient in steady state. In steady state, the heat transfer through a material is proportional to the negative gradient of the temperature and to the area. However, there are some cases in which the Fourier equation is not adequate to describe the heat conduction process. More precisely, Fourier law is diffusive and cannot predict the finite temperature propagation speed in transient situations, in this context, the Cattaneo-Vernotte equation corrects the nonphysical property of infinite propagation of the Fourier and Fickian theory of the diffusion of heat, and this equation also known as the telegraph equation for the temperature is a generalization of the heat diffusion (Fourier's law) and particle diffusion (Fick's laws) equations. Processes where the traditional Fourier heat equation leads to inaccurate temperature and heat flux profiles are known as nonFourier type processes [1]; these processes can be Markovian or non-Markovian [2]. In the Markovian processes case, 
the mean-square displacement of the diffusing particle is proportional to time, while, in the disordered systems or non-Markovian process, the diffusion can be anomalous; in this case the mean-square displacement is proportional to a fractional power of time not equal to one; when anomalous diffusion occurs, the probability density for a diffusing particle is not the usual Gaussian distribution. The mechanism of diffusion is Brownian motion, and this motion is the simplest continuous-time stochastic process. Continuous-time random walks can be coupled with Brownian motion and fractional calculus (FC) to provide an improved estimator in the modeling of anomalous diffusion. A random walk is a mathematical formalization of a path that consists of a succession of random steps [3]. A Lévy flight, also referred to as Lévy motion, is a random walk in which the step-lengths have a heavy-tailed probability distribution. When a random walk is defined as a walk in a space of dimension greater than one, the steps are defined in terms of a probability distribution, and steps move with isotropic random directions [4], and continuous-time random walk schemes are considered in the derivation of time-fractional differential equations. Recently, the subject of FC has attracted interest of researches; this mathematical concept involves nonlocal operators which can be applied in physical systems yielding new information about its behavior, fractional derivatives with respect to coordinates describe power-law nonlocal properties of the distributed system, and there are several papers about the recent history of the FC; see [5-7]. Several approaches have been used for investigating anomalous diffusion, Langevin equations $[8,9]$, random walks $[10,11]$, or fractional derivatives, based on FC several works connected to anomalous diffusion processes which may be found in [12-30]. Using phenomenological arguments, Compte and Metzler [31] generalize the CattaneoVernotte equation by introducing fractional derivatives with a continuous-time random walk scheme. The authors of the works presented in [32-34] studied the generalized CattaneoVernotte equation with fractional space-time derivatives, and the order of the spatial and temporal fractional derivatives are $\beta, \gamma \in(0 ; 2]$. Lewandowska and Kosztołowicz in [35] investigate the subdiffusive impedance phenomena of a spatially limited sample for large pulsation of electric field. Tarasov in [36] based on the Liouville equation obtained the fractional analogues of the classical kinetic and transport equations. Qi and Jiang in [37] derived the exact solution of the Cattaneo-Vernotte equation by joint Laplace and Fourier transforms. Other applications of FC to Cattaneo-Vernotte equation are given in [38-41].

The aim of this work is to contribute to the development of a new version of fractional fundamental Cattaneo-Vernotte equation applying the idea proposed in the work [42]; the order considered is $(0,2]$ for the fractional equation in spacetime domain; this representation preserves the dimensionality of the equation for any value taken by the exponent of the fractional derivative.

The paper is structured as follows: in Section 2 we explain the basic concepts of the fractional calculus; in Section 3 we present the fractional Cattaneo-Vernotte equation and give conclusions in Section 4.

\section{Basic Definitions of Fractional Calculus}

The most commonly used definitions in FC are RiemannLiouville (RL), Grünwald-Letnikov (GL), Caputo fractional derivative (CFD), and Riesz fractional derivative $(R)$ [43-46].

The RL definition of the fractional derivative for $(\varphi>0)$ is

$$
\begin{array}{r}
{ }_{a}^{\mathrm{RL}} D_{t}^{\varphi} f(t)=\frac{1}{\Gamma(m-\varphi)} \frac{d^{m}}{d t^{m}} \int_{a}^{t} \frac{f(\eta)}{(t-\eta)^{\varphi-m+1}} d \eta, \\
m-1<\varphi<m .
\end{array}
$$

For function $f(t)$ the CFD is given by

$$
\begin{array}{r}
{ }_{a}^{C} D_{t}^{\varphi} f(t)=\frac{1}{\Gamma(n-\varphi)} \int_{a}^{t} \frac{f^{(n)}(\eta)}{(-\eta+t)^{\varphi-n+1}} d \eta, \\
n-1<\varphi \leq n,
\end{array}
$$

where $d^{\varphi} / d t^{\varphi}={ }_{a}^{C} D_{t}^{\varphi}$ is a CFD with respect to $t, \varphi \in R$ is the order of the fractional derivative, $n=1,2, \ldots \in N$, and $\Gamma(\cdot)$ represents Euler's gamma function.

In the present paper, we would use the CFD definition, since the former is more popular in real applications. For the CFD definition we need to specify additional conditions in order to produce a unique solution, these additional conditions are expressed in terms of integer-order derivatives [46], and this definition is used mainly for the problem with memories. In the case of the RL definition there exist physically unacceptable initial conditions [47].

The Laplace transform of Caputo's derivative (2) has the form [48]

$$
L\left[{ }_{0}^{C} D_{t}^{\varphi} f(t)\right]=s^{\varphi} F(s)-\sum_{k=0}^{n-1} s^{\varphi-k-1} f^{(k)}(0),
$$

where $F(s)$ is the Laplace transform of the function $f(t)$ and $n=[\Re(\varphi)]+1$. From this expression we have two particular cases:

$$
\begin{aligned}
L\left[{ }_{0}^{C} D_{t}^{\varphi} f(t)\right] & =s^{\varphi} F(s)-s^{\varphi-1} f(0) \quad 0<\varphi \leq 1, \\
L\left[{ }_{0}^{C} D_{t}^{\varphi} f(t)\right] & =s^{\varphi} F(s)-s^{\varphi-1} f(0)-s^{\varphi-2} f^{\prime}(0) \\
& 1<\varphi \leq 2 .
\end{aligned}
$$

The Mittag-Leffler function has gained extensive interest among physicists due its vast potential of applications in the solution of fractional differential equations [48]:

$$
E_{\alpha, \theta}(t)=\sum_{m=0}^{\infty} \frac{t^{m}}{\Gamma(\alpha m+\theta)}, \quad(\alpha>0), \quad(\theta>0) ;
$$

when $\alpha=1$ and $\theta=1$, from (6) we obtain the exponential function.

$\operatorname{erfc}(\alpha)$ denotes the complementary error function [48] and it is defined as

$$
\operatorname{erfc}(\alpha)=\frac{2}{\sqrt{\pi}} \int_{0}^{\alpha} \exp \left(-t^{2}\right) d t
$$



[48]:

Some common Mittag-Leffler functions are described in

$$
\begin{aligned}
& E_{1 / 2,1}( \pm \alpha)=\exp \left(\alpha^{2}\right)[1 \pm \operatorname{erfc}(\alpha)], \\
& E_{1,1}( \pm \alpha)=\exp ( \pm \alpha), \\
& E_{2,1}\left(-\alpha^{2}\right)=\cos (\alpha), \\
& E_{3,1}(\alpha)=\frac{1}{2}\left[\exp \left(\alpha^{1 / 3}\right)\right. \\
& \left.\quad+2 \exp \left(-\left(\frac{1}{2}\right) \alpha^{1 / 3}\right) \cos \left(\frac{\sqrt{3}}{2} \alpha^{1 / 3}\right)\right], \\
& E_{4,1}(\alpha)=\frac{1}{2}\left(\cos \left(\alpha^{1 / 4}\right)+\cosh \left(\alpha^{1 / 4}\right)\right) .
\end{aligned}
$$
[49]:

The Mittag-Leffler function $E_{s / 2, r}$ is defined by Miller in

$$
\begin{aligned}
E_{s / 2, r}(z)= & \frac{z^{2 \kappa(1-r)}}{s} \sum_{j=0}^{s-1} \alpha_{j}^{1-(s / 2+r)}\left(\exp \left(\alpha_{j} z^{2 \kappa}\right)\right) \\
& \cdot\left(\alpha_{j}^{s / 2}+\operatorname{erfc}\left(\alpha_{j}^{1 / 2} z^{\kappa}\right)\right) \\
& -z^{-2 n} \sum_{k=0}^{2 n-1} \frac{z^{k}}{\Gamma(s k / 2+\mu)}
\end{aligned}
$$

where $\kappa=1 / s, r=n s+\mu, n=0,1,2,3, \ldots, \mu=1,2,3, \ldots$. For the calculation of generalized Mittag-Leffler functions at arbitrary precision, see [50,51].

The Riesz fractional derivative for $(\varphi>0)$ is [43-46]

$$
\begin{aligned}
& { }^{R} D_{x}^{\varphi} f(x)=-\frac{1}{2 \cos (\pi \varphi / 2)} \\
& \cdot\left[\frac{1}{\Gamma(n-\varphi)}\left(\frac{\partial}{\partial x}\right)^{n} \int_{-} \infty^{x} \frac{f(\chi, t) d \chi}{(x-\chi)^{\varphi+1-n}}\right. \\
& \left.+\frac{(-1)^{n}}{\Gamma(n-\varphi)}\left(\frac{\partial}{\partial x}\right)^{n} \int_{x}^{\infty} \frac{f(\chi, t) d \chi}{(\chi-x)^{\varphi+1-n}}\right], \\
& n-1<\varphi \leq n,
\end{aligned}
$$

where $d^{\varphi} / d x^{\varphi}={ }^{R} D_{x}^{\varphi}$ is a Riesz fractional derivative with respect to $x, \varphi \in R$ is the order of the fractional derivative, $n=1,2, \ldots \in N$, and $\Gamma(\cdot)$ represents Euler's gamma function.

\section{Fractional Cattaneo-Vernotte Equation}

In previous studies of the fractional Cattaneo-Vernotte equation the authors did not consider the physical dimensionality of the solutions. The authors of the work [42] proposed a systematic way to construct fractional differential equations for the physical systems. To keep the dimensionality of the fractional differential equations a new parameter $\sigma$ was introduced in the following way:

$$
\begin{aligned}
& \frac{\partial}{\partial x} \longrightarrow \frac{1}{\sigma_{x}^{1-\varphi}} \cdot \frac{\partial^{\varphi}}{\partial x^{\varphi}}, \\
& n-1<\varphi \leq n, n \in N=1,2,3, \ldots, \\
& \frac{\partial}{\partial t} \longrightarrow \frac{1}{\sigma_{t}^{1-\varphi}} \cdot \frac{\partial^{\varphi}}{\partial t^{\varphi}}, \\
& n-1<\varphi \leq n, n \in N=1,2,3, \ldots,
\end{aligned}
$$

where $\varphi$ is an arbitrary parameter which represents the order of the derivative, $\sigma_{x}$ has dimension of length, and $\sigma_{t}$ has the dimension of time. These new parameters maintain the dimensionality of the equation invariant and characterizes the fractional space or fractional temporal structures (components that show an intermediate behavior between a conservative system and dissipative one) [42]; when $\varphi=1$ the expressions (15) and (16) reduce to the ordinary derivative. In the following we will apply this idea to generalize the case of the fractional Cattaneo-Vernotte equation.

In this work, we consider generalized Cattaneo-Vernotte equation in the $x$ direction of the form [32-35]

$$
\nabla^{2} T-\frac{1}{D} \dot{T}-\frac{\tau}{D} \ddot{T}=0,
$$

where $\tau$ is a characteristic relaxation time constant (or the non-Fourier character of the material) and $D$ is the generalized thermal diffusivity; (17) is a hyperbolic diffusion equation; when the parameter $\tau=0$, (17) recovers a parabolic form; in this limit, one has to replace Cattaneo-Vernotte equation by Fourier's heat transfer equation.

Considering the CFD (2) and (15) and (16), the fractional representation of (17) is

$$
\begin{gathered}
\frac{1}{\sigma_{x}^{2(1-\varphi)}}{ }_{0}^{C} D_{x}^{2 \varphi} T(x, t)-\frac{1}{D} \cdot \frac{1}{\sigma_{t}^{1-\varphi}}{ }_{0}^{C} D_{t}^{\varphi} T(x, t)-\frac{\tau}{D} \\
\cdot \frac{1}{\sigma_{t}^{2(1-\varphi)}}{ }_{0}^{C} D_{t}^{2 \varphi} T(x, t)=0 .
\end{gathered}
$$

The order of the derivative considered is $\varphi \in(0,2]$ for the fractional Cattaneo-Vernotte equation in space-time domain.

3.1. Fractional Space Cattaneo-Vernotte Equation. Considering (18) and assuming that the space derivative is fractional equation (15) and the time derivative is ordinary, the spatial fractional equation is

$$
\begin{gathered}
{ }_{0}^{C} D_{x}^{2 \varphi} T(x, t)-\frac{1}{D} \sigma_{x}^{2(1-\varphi)} \cdot \frac{\partial T(x, t)}{\partial t}-\frac{\tau}{D} \sigma_{x}^{2(1-\varphi)} \\
\cdot \frac{\partial^{2} T(x, t)}{\partial t^{2}}=0 .
\end{gathered}
$$

Suppose the solution

$$
T(x, t)=T_{0} \cdot \exp (i \omega t) u(x) ;
$$


substituting (20) into (19) we obtain

$$
\frac{d^{2 \varphi} u(x)}{d x^{2 \varphi}}+\left(\frac{\tau}{D} \omega^{2}-i \frac{1}{D} \omega\right) \sigma_{x}^{2(1-\varphi)} u(x)=0,
$$

where

$$
k_{x}^{2}=\left(\frac{\tau}{D} \omega^{2}-i \frac{1}{D} \omega\right)
$$

is the dispersion relation in the $x$ direction and

$$
\widetilde{k}_{x}^{2}=\left(\frac{\tau}{D} \omega^{2}-i \frac{1}{D} \omega\right) \sigma_{x}^{2(1-\varphi)}=k_{x}^{2} \sigma_{x}^{2(1-\varphi)},
$$

is the fractional dispersion relation; from the fractional dispersion relation (23), we can expect the fractional wave number $\widetilde{k}$ in the $x$ direction to have real and imaginary parts, $\delta_{x}$ and $\beta_{x}$, respectively. Let us write

$$
\tilde{k}_{x}=\delta_{x}-i \beta_{x}
$$

substituting (24) into (23) we have

$$
\left(\delta_{x}-i \beta_{x}\right)^{2}=\delta_{x}^{2}-2 i \delta_{x} \beta_{x}-\beta_{x}^{2}
$$

where

$$
\delta_{x}^{2}-2 i \delta_{x} \beta_{x}-\beta_{x}^{2}=\left(\frac{\tau}{D} \omega^{2}-i \frac{1}{D} \omega\right) \sigma_{x}^{2(1-\varphi)} ;
$$

solving for $\beta_{x}$ we obtain

$$
\beta_{x}=\frac{\omega}{D} \cdot \frac{1}{2 \delta_{x}} \sigma_{x}^{2(1-\varphi)},
$$

and for $\delta_{x}$

$$
\delta_{x}=\omega \sqrt{\frac{\tau}{D}}\left[\frac{1}{2} \pm \frac{1}{2} \sqrt{1+\frac{1}{\tau^{2} \omega^{2}}}\right]^{1 / 2} \sigma_{x}^{1-\varphi}
$$

substituting (28) into (27) we have

$$
\beta_{x}=\frac{1}{2 D \sqrt{\tau / D}\left[1 / 2 \pm(1 / 2) \sqrt{1+1 / \tau^{2} \omega^{2}}\right]^{1 / 2}} \sigma_{x}^{1-\varphi} .
$$

Now the fractional wave number is $\tilde{k}_{x}=\delta_{x}-i \beta_{x}$, where $\delta_{x}$ and $\beta_{x}$ are given by (28) and (29), respectively,

$$
\begin{aligned}
& \tilde{k}_{x} \\
& \begin{array}{c}
=\omega \sqrt{\frac{\tau}{D}\left[\frac{1}{2} \pm \frac{1}{2} \sqrt{1+\frac{1}{\tau^{2} \omega^{2}}}\right]^{1 / 2} \sigma_{x}^{1-\varphi}} \\
-i \frac{1}{2 D \sqrt{\tau / D}\left[1 / 2 \pm(1 / 2) \sqrt{1+1 / \tau^{2} \omega^{2}}\right]^{1 / 2}} \sigma_{x}^{1-\varphi}
\end{array}
\end{aligned}
$$

equation (30) describes the real and imaginary part of the fractional wave number in terms of the frequency $\omega$, the relaxation time $\tau$, and the generalized thermal diffusivity $D$, in presence of fractional space components $\sigma_{x}$.
Considering (23), (21) gives

$$
\frac{d^{2 \varphi} u(x)}{d x^{2 \varphi}}+\widetilde{k}_{x}^{2} u(x)=0,
$$

the solution of (31) can be obtained applying direct and inverse Laplace transform [47], and the solution of the above equation is given by

$$
u(x)=E_{2 \varphi, 1}\left(-\tilde{k}_{x}^{2} x^{2 \varphi}\right)
$$

where $E_{2 \varphi, 1}\left(-\widetilde{k}_{x}^{2} x^{2 \varphi}\right)$ is the Mittag-Leffler function.

Therefore the general solution of (21) is given by

$$
T(x, t)=T_{0} \cdot \exp (i \omega t) \cdot E_{2 \varphi, 1}\left(-\tilde{k}_{x}^{2} x^{2 \varphi}\right)
$$

Next, we will analyze the case when $\varphi$ takes different values.

Case 1. When $\varphi=2$, we have

$$
\begin{aligned}
\tilde{k}_{x}= & \omega \sqrt{\frac{\tau}{D}}\left[\frac{1}{2} \pm \frac{1}{2} \sqrt{1+\frac{1}{\tau^{2} \omega^{2}}}\right]^{1 / 2} \sigma_{x}^{-1} \\
& -i \frac{1}{2 D \sqrt{\tau / D}\left[1 / 2 \pm(1 / 2) \sqrt{1+1 / \tau^{2} \omega^{2}}\right]^{1 / 2}} \sigma_{x}^{-1}
\end{aligned}
$$

and equation (34) represents the fractional wave number in presence of fractional space components $\sigma_{x}$.

In this case, (33) is written as follows:

$$
T(x, t)=T_{0} \cdot \exp (i \omega t) \cdot E_{4,1}\left(-\tilde{k}_{x}^{2} x^{4}\right)
$$

where $E_{4,1}$ is given by (12) and solution (35) is

$$
\begin{aligned}
T(x, t)= & \frac{T_{0}}{2} \cdot \exp (i \omega t) \\
& \cdot\left[\cos \left(-\tilde{k}_{x}^{1 / 2} x\right)+\cosh \left(-\tilde{k}_{x}^{1 / 2} x\right)\right] .
\end{aligned}
$$

Case 2. When $\varphi=3 / 2$, we have

$$
\begin{aligned}
\tilde{k}_{x} & \\
= & \omega \sqrt{\frac{\tau}{D}}\left[\frac{1}{2} \pm \frac{1}{2} \sqrt{1+\frac{1}{\tau^{2} \omega^{2}}}\right]^{1 / 2} \sigma_{x}^{-1 / 2} \\
& -i \frac{1}{2 D \sqrt{\tau / D}\left[1 / 2 \pm(1 / 2) \sqrt{1+1 / \tau^{2} \omega^{2}}\right]^{1 / 2}} \sigma_{x}^{-1 / 2},
\end{aligned}
$$

and equation (37) represents the fractional wave number in presence of fractional space components $\sigma_{x}$.

In this case, (33) is written as follows:

$$
T(x, t)=T_{0} \cdot \exp (i \omega t) \cdot E_{3,1}\left(-\widetilde{k}_{x}^{2} x^{3}\right),
$$


where $E_{3,1}$ is given by (11) and solution (38) is

$$
\begin{aligned}
& T(x, t)=\frac{T_{0}}{2} \cdot \exp (i \omega t) \cdot\left[\exp \left(-\tilde{k}_{x}^{2 / 3} x\right)\right. \\
& \left.\quad+2 \exp \left(\frac{\widetilde{k}_{x}^{2 / 3}}{2} x\right) \cdot \cos \left(-\frac{\sqrt{3}}{2} \tilde{k}_{x}^{2 / 3} x\right)\right] .
\end{aligned}
$$

Case 3. When $\varphi=1$, we have $\tilde{k}_{x}=k_{x}$

$$
\begin{aligned}
k_{x}= & \omega \sqrt{\frac{\tau}{D}}\left[\frac{1}{2} \pm \frac{1}{2} \sqrt{1+\frac{1}{\tau^{2} \omega^{2}}}\right]^{1 / 2} \\
& -i \frac{1}{2 D \sqrt{\tau / D}\left[1 / 2 \pm(1 / 2) \sqrt{1+1 / \tau^{2} \omega^{2}}\right]^{1 / 2}}
\end{aligned}
$$

and equation (40) represents the classical wave number $k_{x}$. From (33) we have

$$
T(x, t)=T_{0} \cdot \exp (i \omega t) \cdot E_{2,1}\left(-\widetilde{k}_{x}^{2} x^{2}\right),
$$

where

$$
T(x, t)=\Re\left[T_{0} \cdot \exp (i \omega t) \cdot \exp \left(-i \tilde{k}_{x} x\right)\right],
$$

and, in (42), $\tilde{k}_{x}=k_{x}, \mathfrak{R}$ indicates the real part, and $k_{x}=$ $\delta_{x}-i \beta_{x}$ is wave number (40); substituting $k_{x}$ in (42) we have

$$
T(x, t)=\mathfrak{R}\left[T_{0} \cdot \exp \left(i\left(\omega t-\delta_{x} x\right)\right) \cdot \exp \left(-\beta_{x} x\right)\right] .
$$

Equation (43) represents the classical case for the space Cattaneo-Vernotte equation. The first exponential exp $(i(\omega t-$ $\left.\delta_{x} x\right)$ ) gives the usual plane-wave variation of the thermal field with position $x$ and time $t$. The second exponential $\exp \left(-\beta_{x} x\right)$ gives and exponential decay in the amplitude of the thermal wave.

Case 4. When $\varphi=1 / 2$, from (33) we have

$$
T(x, t)=T_{0} \cdot \exp (i \omega t) \cdot E_{1,1}\left(-\tilde{k}_{x}^{2} x\right),
$$

where $\tilde{k}_{x}$ is

$$
\begin{aligned}
\tilde{k}_{x}= & \omega \sqrt{\frac{\tau}{D}}\left[\frac{1}{2} \pm \frac{1}{2} \sqrt{1+\frac{1}{\tau^{2} \omega^{2}}}\right]^{1 / 2} \sigma_{x}^{1 / 2} \\
& -i \frac{1}{2 D \sqrt{\tau / D}\left[1 / 2 \pm(1 / 2) \sqrt{1+1 / \tau^{2} \omega^{2}}\right]^{1 / 2}} \sigma_{x}^{1 / 2},
\end{aligned}
$$

and equation (45) represents the fractional wave number in presence of fractional space components $\sigma_{x}$.

The solution for (44) is

$$
\begin{aligned}
& T(x, t)=\mathfrak{R}\left[T_{0} \cdot \exp \left(i \omega\left(t+\frac{1}{D} \sigma_{x} x\right)\right)\right. \\
& \left.\cdot \exp \left(-\frac{\tau}{D} \omega^{2} \sigma_{x} x\right)\right],
\end{aligned}
$$

where $\mathfrak{R}$ indicates the real part.
Case 5. When $\varphi=1 / 4$, from (33) we have

$$
T(x, t)=T_{0} \cdot \exp (i \omega t) \cdot E_{1 / 2,1}\left(-\tilde{k}_{x}^{2} x^{1 / 2}\right),
$$

where $\tilde{k}_{x}$ is

$$
\begin{aligned}
\tilde{k}_{x}= & \omega \sqrt{\frac{\tau}{D}}\left[\frac{1}{2} \pm \frac{1}{2} \sqrt{1+\frac{1}{\tau^{2} \omega^{2}}}\right]^{1 / 2} \sigma_{x}^{3 / 4} \\
& -i \frac{1}{2 D \sqrt{\tau / D}\left[1 / 2 \pm(1 / 2) \sqrt{1+1 / \tau^{2} \omega^{2}}\right]^{1 / 2}} \sigma_{x}^{3 / 4},
\end{aligned}
$$

and equation (48) represents the fractional wave number in presence of fractional space components $\sigma_{x}$.

In this case, (33) is written as follows:

$$
T(x, t)=T_{0} \cdot \exp (i \omega t) \cdot E_{1 / 2,1}\left(-\tilde{k}_{x}^{2} x^{1 / 2}\right)
$$

where $E_{1 / 2,1}$ is given by (8) and solution (49) is

$$
\begin{aligned}
T(x, t)= & T_{0} \cdot \exp (i \omega t) \cdot \exp \left(\widetilde{k}^{4} x\right) \\
& \cdot\left[1-\operatorname{erfc}\left(-\widetilde{k}^{2} x^{1 / 2}\right)\right]
\end{aligned}
$$

$\operatorname{erfc}(\alpha)$ denotes the error function defined in (8). Equation (50) represents the space evolution of the temperature and the amplitude exhibits an algebraic decay for $x \rightarrow \infty$.

For this case there exists a physical relation between the auxiliary parameter $\sigma_{x}$ and the wave number $k_{x}$ given by the order $\varphi$ of the fractional differential equation

$$
\varphi=k_{x} \sigma_{x}=\frac{\sigma_{x}}{\lambda}, \quad 0<\sigma_{x} \leq \lambda,
$$

where $\lambda$ is the wavelength. We can use this relation in order to write (33) as

$$
T(\tilde{x}, t)=T_{0} \cdot \exp (i \omega t) \cdot E_{2 \varphi}\left(-\varphi^{2(1-\varphi)} \tilde{x}^{2 \varphi}\right),
$$

where $\tilde{x}=x / \lambda$ is a dimensionless parameter. Figures 1 (a) and (b) show the simulation of (52) for $\varphi$ values $0.7<\varphi \leq 1$ and $1.7<\varphi \leq 2$, respectively.

Table 1 shows the different solutions of (52). The order of the fractional differential equation is $\varphi=2, \varphi=3 / 2, \varphi=1$, $\varphi=1 / 2$, and $\varphi=1 / 4$.

3.2. Fractional Time Cattaneo-Vernotte Equation. Considering (18) and assuming that the time derivative is fractional equation (16) and the space derivative is ordinary, the temporal fractional equation is

$$
\begin{gathered}
{ }_{0}^{C} D_{t}^{2 \varphi} T(x, t)+\frac{1}{\tau} \cdot \sigma_{t}^{1-\varphi C}{ }_{0}^{\varphi} D_{t}^{\varphi} T(x, t) \\
-\frac{D}{\tau} \sigma_{t}^{2(1-\varphi)} \frac{\partial^{2} T(x, t)}{\partial x^{2}}=0
\end{gathered}
$$




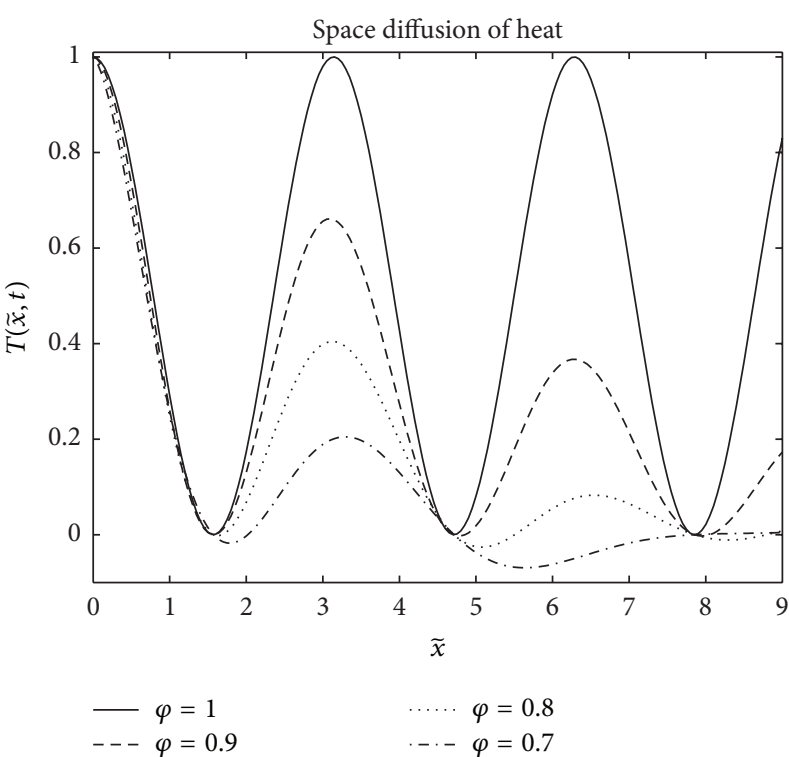

(a)

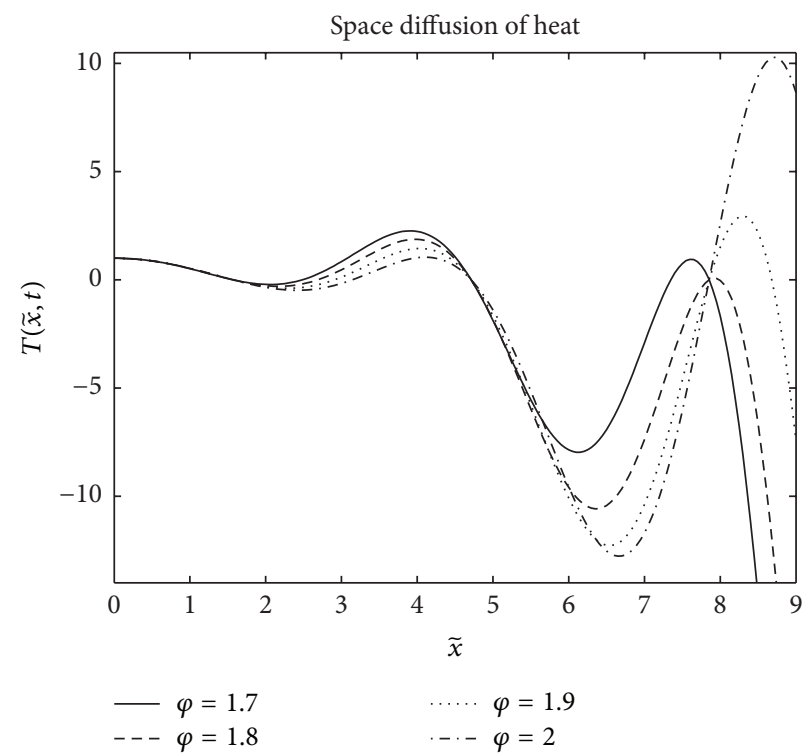

(b)

FIGURE 1: Simulation of (52) for $0.7<\varphi \leq 2$.

TABLE 1: Solutions for fractional space Cattaneo-Vernotte equation (52) for different values of $\varphi ; \Re$ indicates the real part and $\operatorname{erfc}(\alpha)$ denotes the error function defined in (8).

\begin{tabular}{ll}
\hline$\varphi$ & Solution \\
\hline 2 & $T(x, t)=\frac{T_{0}}{2} \cdot \exp (i \omega t) \cdot\left[\cos \left(-\tilde{k}_{x}^{1 / 2} x\right)+\cosh \left(-\tilde{k}_{x}^{1 / 2} x\right)\right]$ \\
\hline $3 / 2$ & $T(x, t)=\frac{T_{0}}{2} \cdot \exp (i \omega t) \cdot\left[\exp \left(-\tilde{k}_{x}^{2 / 3} x\right)\right.$ \\
& $\left.+2 \exp \left(\frac{\tilde{k}_{x}^{2 / 3}}{2} x\right) \cdot \cos \left(-\frac{\sqrt{3}}{2} \widetilde{k}_{x}^{2 / 3} x\right)\right]$ \\
\hline $1 / 2$ & $T(x, t)=\mathfrak{R}\left[T_{0} \cdot \exp \left(i \omega\left(t+\frac{1}{D} \sigma_{x} x\right)\right) \cdot \exp \left(-\frac{\tau}{D} \omega^{2} \sigma_{x} x\right)\right]$ \\
\hline $1 / 4$ & $T(x, t)=T_{0} \cdot \exp (i \omega t) \cdot \exp \left(\widetilde{k}^{4} x\right) \cdot\left[1-\operatorname{erfc}\left(-\tilde{k}^{2} x^{1 / 2}\right)\right]$ \\
\hline
\end{tabular}

suppose the solution

$$
T(x, t)=T_{0} \exp \left(i \widetilde{k}_{x} x\right) u(t),
$$

where $\widetilde{k}_{x}$ is the wave number in the $x$ direction. Substituting (54) into (53) we obtain

$$
\frac{d^{2 \varphi} u(t)}{d t^{2 \varphi}}+\frac{1}{\tau} \sigma_{t}^{1-\varphi} \frac{d^{\varphi} u(t)}{d t^{\varphi}}+\frac{D}{\tau} \widetilde{k}_{x}^{2} \sigma_{t}^{2(1-\varphi)} u(t)=0
$$

the solution of (55) can be obtained applying direct and inverse Laplace transform [47]. Taking solution (54) we have

$$
\begin{aligned}
T(x, t)= & T_{0} \cdot \exp \left(i \widetilde{k}_{x} x\right) \cdot E_{\varphi, 1}\left(-\frac{1}{2 \tau} \sigma_{t}^{1-\varphi} t^{\varphi}\right) \\
& \cdot E_{2 \varphi, 1}\left(-\left[\frac{D}{\tau} \widetilde{k}_{x}^{2}-\frac{1}{4 \tau^{2}}\right] \sigma_{t}^{2(1-\varphi)} t^{2 \varphi}\right),
\end{aligned}
$$

and solution (56) represents a temporal nonlocal thermal equation interpreted as an existence of memory effects which correspond to intrinsic dissipation characterized by the exponent of the fractional derivative $\varphi$ in the system.

For underdamped case, we have $\left((D / \tau) \widetilde{k}_{x}^{2}-1 / 4 \tau^{2}\right)=0$, $\omega_{0}=\sqrt{D / \tau} \widetilde{k}_{x}$ is the undamped natural frequency expressed in radians per second, and $\alpha=\sqrt{1 / 2 \tau}$ is the damping factor expressed in meters per second. Next, we will analyze the case when $\varphi$ takes different values.

Case 1. When $\varphi=2$, from (56) we have

$$
\begin{aligned}
T(x, t)= & T_{0} \cdot \exp \left(i \tilde{k}_{x} x\right) \cdot E_{2,1}\left(-\frac{1}{2 \tau \sigma_{t}} t^{2}\right) \\
& \cdot E_{4,1}\left(-\left[\frac{D}{\tau} \widetilde{k}_{x}^{2}-\frac{1}{4 \tau^{2}}\right]\left(\frac{1}{\sigma_{t}^{2}}\right) t^{4}\right),
\end{aligned}
$$

where $E_{2,1}$ is given by (10) and $E_{4,1}$ by (12); in this case solution (57) is

$$
\begin{gathered}
T(x, t)=\frac{T_{0}}{2} \cdot \exp \left(i \widetilde{k}_{x} x\right) \cdot \cos \left(\sqrt{\frac{1}{2 \sigma_{t} \tau}} t\right) \\
.\left\{\cos \left[-\left(\frac{D}{\tau} \widetilde{k}_{x}^{2}-\frac{1}{4 \tau^{2}}\right)\left(\frac{1}{\sigma_{t}^{2}}\right)\right]^{1 / 4} t\right. \\
\left.+\cosh \left[-\left(\frac{D}{\tau} \widetilde{k}_{x}^{2}-\frac{1}{4 \tau^{2}}\right)\left(\frac{1}{\sigma_{t}^{2}}\right)\right]^{1 / 4} t\right\} .
\end{gathered}
$$


Case 2. When $\varphi=3 / 2$, from (56) we have

$$
\begin{aligned}
T(x, t)= & T_{0} \cdot \exp \left(i \tilde{k}_{x} x\right) \cdot E_{3 / 2,1}\left(-\frac{1}{2 \tau \sigma_{t}^{1 / 2}} t^{3 / 2}\right) \\
& \cdot E_{3,1}\left(-\left[\frac{D}{\tau} \tilde{k}_{x}^{2}-\frac{1}{4 \tau^{2}}\right]\left(\frac{1}{\sigma_{t}}\right) t^{3}\right),
\end{aligned}
$$

where $E_{3 / 2,1}$ is given by (13) and $E_{3,1}$ by (11); in this case solution (59) is

$$
\begin{aligned}
& T(x, t)=\frac{T_{0}}{2} \cdot \exp \left(i \tilde{k}_{x} x\right) \\
& {\left[\frac{\left(-t^{3 / 2} / 2 \tau \sigma_{t}^{1 / 2}\right)^{2 \kappa(1-r)}}{3} \sum_{j=0}^{2} \alpha_{j}^{1-(3 / 2+r)}\right.} \\
& \cdot\left(\exp \left(\alpha_{j}\left(-\frac{t^{3 / 2}}{2 \tau \sigma_{t}^{1 / 2}}\right)^{2 \kappa}\right)\right) \\
& \cdot\left(\alpha_{j}^{3 / 2}+\operatorname{erfc}\left(\alpha_{j}^{1 / 2}\left(-\frac{t^{3 / 2}}{2 \tau \sigma_{t}^{1 / 2}}\right)^{\kappa}\right)\right) \\
& \left.-\left(-\frac{t^{3 / 2}}{2 \tau \sigma_{t}^{1 / 2}}\right)^{-2 n} \cdot \sum_{k=0}^{2 n-1} \frac{\left(-t^{3 / 2} / 2 \tau \sigma_{t}^{1 / 2}\right)^{k}}{\Gamma(3 k / 2+\mu)}\right] \\
& {\left[\exp \left(-\left[\frac{D}{\tau} \widetilde{k}_{x}^{2}-\frac{1}{4 \tau^{2}}\right]^{2 / 3}\left(\frac{1}{\sigma_{t}^{2 / 3}}\right) t\right)\right.} \\
& +2 \exp \left(\frac{\left[\left((D / \tau) \tilde{k}_{x}^{2}-1 / 4 \tau^{2}\right)\left(1 / \sigma_{t}\right)\right]^{2 / 3}}{2} t\right) \\
& \left.\cdot \cos \left(-\frac{\sqrt{3}}{2}\left[\left(\frac{D}{\tau} \widetilde{k}_{x}^{2}-\frac{1}{4 \tau^{2}}\right)\left(\frac{1}{\sigma_{t}}\right)\right]^{2 / 3} t\right)\right],
\end{aligned}
$$

where $\kappa=1 / 3, r=3 n+\mu, n=0,1,2,3, \ldots, \mu=1,2,3, \ldots$.

Case 3. When $\varphi=1$, from (56) we have

$$
\begin{aligned}
T(x, t)= & T_{0} \cdot \exp \left(i \tilde{k}_{x} x\right) \cdot \exp \left(-\frac{t}{2 \tau}\right) \\
& \cdot \cos \left(\sqrt{\frac{D}{\tau} \widetilde{k}_{x}^{2}-\frac{1}{4 \tau^{2}}} t\right),
\end{aligned}
$$

and (61) represents the classic case and the well-known result; from (61) we see that there is a relation between $\varphi$ and $\sigma_{t}$ given by

$$
\begin{aligned}
\varphi=\left(\frac{D}{\tau} \widetilde{k}_{x}^{2}-\frac{1}{4 \tau^{2}}\right)^{1 / 2} & \sigma_{t} \\
& 0<\sigma_{t} \leq \frac{1}{\left((D / \tau) \tilde{k}_{x}^{2}-1 / 4 \mu^{2}\right)^{1 / 2}} .
\end{aligned}
$$

Then solution (56) for the underdamped case $\alpha<\omega_{0}$ takes the form

$$
\begin{aligned}
T(x, t)= & T_{0} \cdot \exp \left(i \tilde{k}_{x} x\right) \\
& \cdot E_{\varphi, 1}\left(-\frac{1}{2 \tau \sqrt{(D / \tau) \widetilde{k}_{x}^{2}-1 / 4 \tau^{2}}} \varphi^{1-\varphi \widetilde{t}^{\varphi}}\right) \\
& \cdot E_{2 \varphi, 1}\left(-\varphi^{2(1-\varphi)} t^{2 \varphi}\right),
\end{aligned}
$$

where $\widehat{t}=\left((D / \tau) \widetilde{k}_{x}^{2}-1 / 4 \tau^{2}\right)^{1 / 2} t$ is a dimensionless parameter.

Due to the condition $\alpha<\omega_{0}$ we can choose an example

$$
\begin{aligned}
\frac{1}{2 \tau \sqrt{(D / \tau) \tilde{k}_{x}^{2}-1 / 4 \tau^{2}}} & =3, \\
0 & \leq \frac{1}{2 \tau \sqrt{(D / \tau) \tilde{k}_{x}^{2}-1 / 4 \tau^{2}}}<\infty .
\end{aligned}
$$

So, solution (56) takes its final form:

$$
\begin{aligned}
T(x, t)= & T_{0} \cdot \exp \left(i \widetilde{k}_{x} x\right) \cdot E_{\varphi, 1}\left(-3 \varphi^{1-\varphi \widehat{t}^{\varphi}}\right) \\
& \cdot E_{2 \varphi, 1}\left(-\varphi^{2(1-\varphi)} \hat{t}^{2 \varphi}\right) .
\end{aligned}
$$

Case 4. When $\varphi=1 / 2$, from (56) we have

$$
\begin{aligned}
T(x, t)= & T_{0} \cdot \exp \left(i \widetilde{k}_{x} x\right) \cdot \exp \left(\frac{\sigma_{t}}{4 \tau^{2}} t\right) \\
& \cdot\left[1-\operatorname{erfc}\left(-\frac{\sigma_{t}^{1 / 2}}{2 \tau} t^{1 / 2}\right)\right] \\
& \cdot \exp \left[-\left(\frac{D}{\tau} \widetilde{k}_{x}^{2}-\frac{1}{4 \tau^{2}}\right) \sigma_{t} t\right],
\end{aligned}
$$

and $\operatorname{erfc}(\alpha)$ denotes the error function defined in (8). Equation (66) represents the time evolution of the temperature and the amplitude exhibits an algebraic decay for $t \rightarrow \infty$. Plots for different values of $\varphi$ are shown in Figures 2(a) and 2(b).

Table 2 shows the different solutions of (65). The order of the fractional differential equation is $\varphi=2, \varphi=3 / 2$, $\varphi=1$, and $\varphi=1 / 2$. The change of the order of the derivative describes the crossover from ballistic transport to the diffusion behavior.

In the overdamped case, $\alpha>\omega_{0}$ or $\eta>2 \widetilde{k}_{x} \sqrt{\epsilon / \mu}$, the solution of (56) has the form

$$
\begin{aligned}
T(x, t)= & T_{0} \cdot \exp \left(i \tilde{k}_{x} x\right) \cdot E_{\varphi, 1}\left(-\frac{\sigma_{t}^{1-\varphi}}{2 \tau} t^{\varphi}\right) \\
& \cdot E_{\varphi, 1}\left(-\left[\frac{1}{4 \tau^{2}}-\frac{D}{\tau} \widetilde{k}_{x}^{2}\right]^{1 / 2} \sigma_{t}^{1-\varphi} t^{\varphi}\right) .
\end{aligned}
$$

Next, we will analyze the case when $\varphi$ takes different values. 


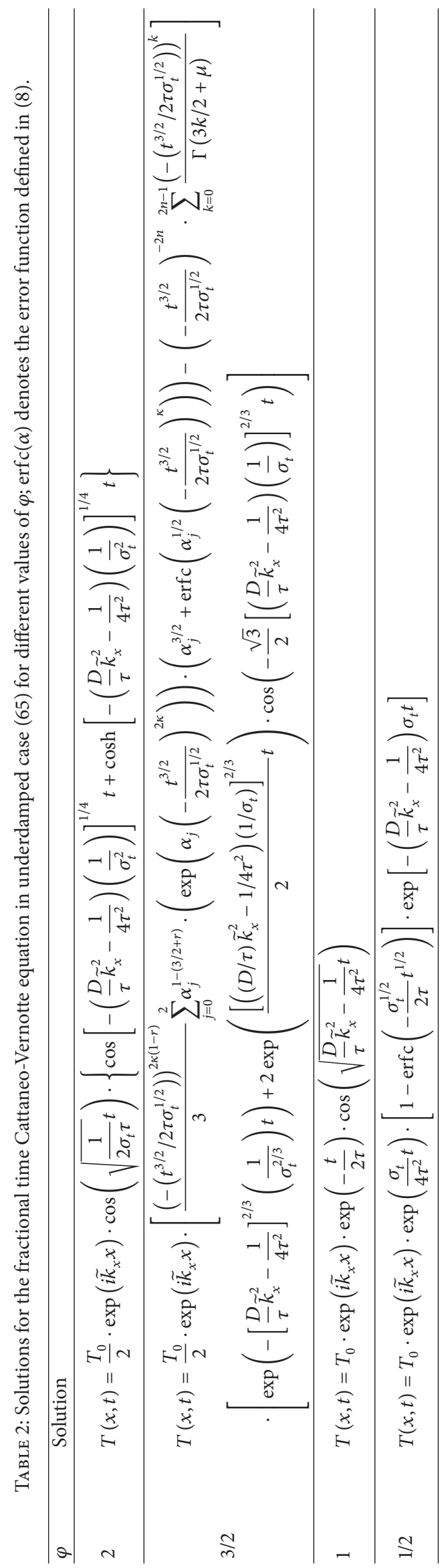




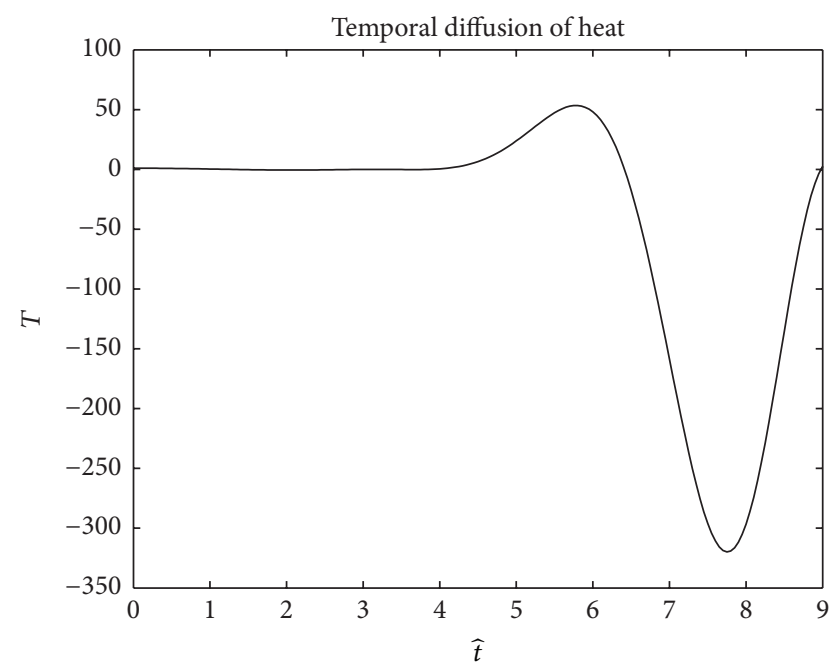

$-\varphi=2$

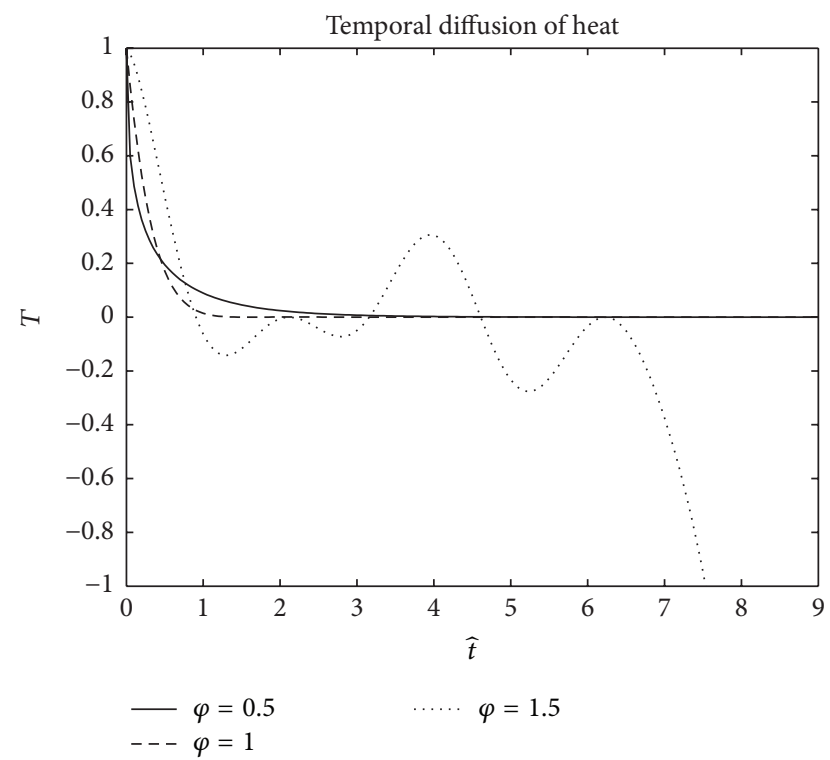

(b)

FIGURE 2: Simulation of (65) for $0.5<\varphi \leq 2$. The crossover time occurs at $\widehat{t}=1.0$ independent of $\varphi$, but the crossover time increases with decreasing $\varphi$.

Case 1. When $\varphi=2$, we have

$$
\begin{aligned}
T(x, t)= & T_{0} \cdot \exp \left(i \widetilde{k}_{x} x\right) \cdot E_{2,1}\left(-\frac{\sigma_{t}^{-1}}{2 \tau} t^{2}\right) \\
& \cdot E_{2,1}\left(-\left[\frac{1}{4 \tau^{2}}-\frac{D}{\tau} \widetilde{k}_{x}^{2}\right]^{1 / 2} \sigma_{t}^{-1} t^{2}\right),
\end{aligned}
$$

$$
\cdot \cos \left(\sqrt{\left(\frac{1}{4 \tau^{2}}-\frac{D}{\tau} k_{x}^{2}\right)^{1 / 2}\left(\frac{1}{\sigma_{t}}\right) t}\right) \text {. }
$$

Case 2. When $\varphi=3 / 2$, we have

$$
\begin{aligned}
T(x, t)= & T_{0} \cdot \exp \left(i \tilde{k}_{x} x\right) \cdot E_{3 / 2,1}\left(-\frac{1}{2 \tau \sigma_{t}^{-1 / 2}} t^{3 / 2}\right) \\
& \cdot E_{3 / 2,1}\left(-\left[\frac{1}{4 \tau^{2}}-\frac{D}{\tau} \widetilde{k}_{x}^{2}\right]^{1 / 2} \sigma_{t}^{-1 / 2} t^{3 / 2}\right),
\end{aligned}
$$

where $E_{3 / 2,1}$ is given by (13); in this case solution (70) is

$$
\begin{gathered}
T(x, t)=\frac{T_{0}}{2} \cdot \exp \left(i \widetilde{k}_{x} x\right) \cdot\left[\frac{\left(-t^{3 / 2} / 2 \tau \sigma_{t}^{1 / 2}\right)^{2 \kappa(1-r)}}{3}\right. \\
\cdot \sum_{j=0}^{2} \alpha_{j}^{1-(3 / 2+r)}\left(\exp \left(\alpha_{j}\left(-\frac{t^{3 / 2}}{2 \tau \sigma_{t}^{1 / 2}}\right)^{2 \kappa}\right)\right) \cdot\left(\alpha_{j}^{3 / 2}\right.
\end{gathered}
$$

$$
\begin{aligned}
& \left.+\operatorname{erfc}\left(\alpha_{j}^{1 / 2}\left(-\frac{t^{3 / 2}}{2 \tau \sigma_{t}^{1 / 2}}\right)^{\kappa}\right)\right)-\left(-\frac{t^{3 / 2}}{2 \tau \sigma_{t}^{1 / 2}}\right)^{-2 n} \\
& \left.\cdot \sum_{k=0}^{2 n-1} \frac{\left(-t^{3 / 2} / 2 \tau \sigma_{t}^{1 / 2}\right)^{k}}{\Gamma(3 k / 2+\mu)}\right] \\
& \cdot\left[\frac{\left(-\left(1 / 4 \tau^{2}-(D / \tau) \tilde{k}_{x}^{2}\right)^{1 / 2} \sigma_{t}^{-1 / 2} t^{3 / 2}\right)}{3}\right. \\
& \cdot \sum_{j=0}^{2} \alpha_{j}^{1-(3 / 2+r)} \\
& \cdot\left(\exp \left(\alpha_{j}\left(-\left(\frac{1}{4 \tau^{2}}-\frac{D}{\tau} \widetilde{k}_{x}^{2}\right) \sigma_{t}^{-1 / 2} t^{3 / 2}\right)\right)^{2 k}\right)
\end{aligned}
$$$$
\left(\alpha_{j}^{3 / 2}\right.
$$$$
\left.+\operatorname{erfc}\left(\alpha_{j}^{1 / 2}\left(-\left(\frac{1}{4 \tau^{2}}-\frac{D}{\tau} \widetilde{k}_{x}^{2}\right) \sigma_{t}^{-1 / 2} t^{3 / 2}\right)^{k}\right)\right)
$$$$
-\left(-\left(\frac{1}{4 \tau^{2}}-\frac{D}{\tau} \widetilde{k}_{x}^{2}\right)^{1 / 2} \sigma_{t}^{-1 / 2} t^{3 / 2}\right)^{-2 n}
$$$$
\left.\cdot \sum_{k=0}^{2 n-1} \frac{\left(-\left(1 / 4 \tau^{2}-(D / \tau) \tilde{k}_{x}^{2}\right)^{1 / 2} \sigma_{t}^{-1 / 2} t^{3 / 2}\right)^{k}}{\Gamma(3 k / 2+\mu)}\right] \text {, }
$$ 


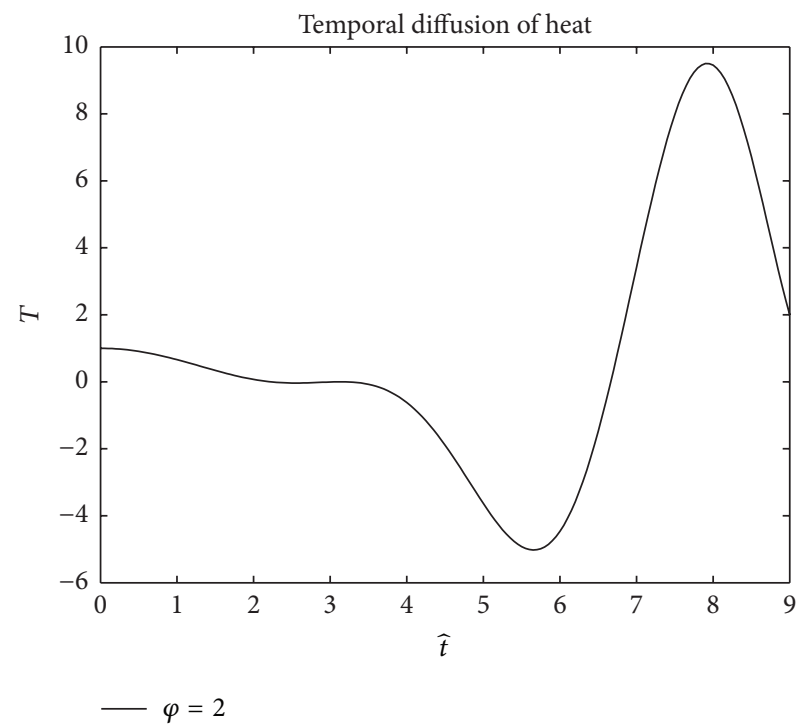

(a)

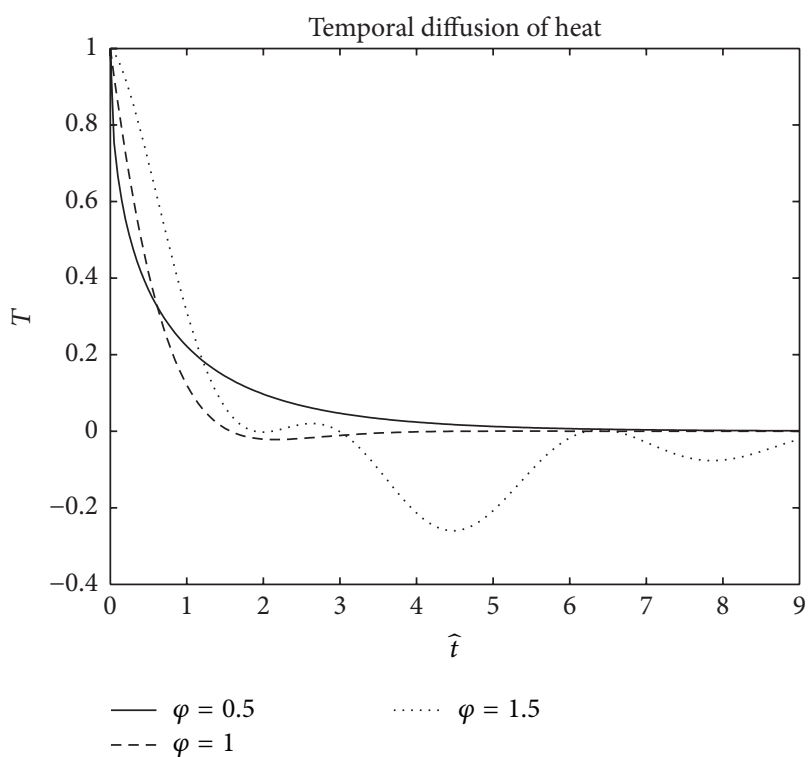

(b)

FIgURE 3: Simulation of (76) for $0.5<\varphi \leq 2$. The crossover time occurs at $\widehat{t}=1.0$ independent of $\varphi$, but the crossover time increases with decreasing $\varphi$.

Case 3. When $\varphi=1$, from (67) we have

$$
\begin{aligned}
T(x, t)= & T_{0} \cdot \exp \left(i \tilde{k}_{x} x\right) \\
& \cdot \exp \left(-\frac{1}{2 \tau}\left(1+\sqrt{1-\frac{4 \tau \tilde{k}_{x}^{2}}{D}}\right) t\right),
\end{aligned}
$$

and solution (72) represents the classic case.

Taking into account, the relation between $\varphi$ and $\sigma_{t}$ is

$$
\begin{aligned}
\varphi=\left(\frac{1}{4 \tau^{2}}-\frac{D}{\tau} \widetilde{k}_{x}^{2}\right)^{1 / 2} & \sigma_{t} \\
& 0<\sigma_{t} \leq \frac{1}{\left(1 / 4 \tau^{2}-(D / \tau) \tilde{k}_{x}^{2}\right)^{1 / 2}}
\end{aligned}
$$

Solution (67) takes the form

$$
\begin{aligned}
T(x, t)= & T_{0} \cdot \exp \left(i \widetilde{k}_{x} x\right) \\
& \cdot E_{\varphi, 1}\left(-\frac{1}{2 \tau \sqrt{1 / 4 \tau^{2}-(D / \tau) \tilde{k}_{x}^{2}}} \varphi^{1-\varphi} t^{\varphi}\right) \\
& \cdot E_{\varphi, 1}\left(-\varphi^{1-\varphi} t^{\varphi}\right)
\end{aligned}
$$

where $\widehat{t}=\left(1 / 4 \tau^{2}-(D / \tau) \tilde{k}_{x}^{2}\right)^{1 / 2} t$ is a dimensionless parameter.
Due to the condition $\alpha>\omega_{0}$ we can choose an example

$$
\begin{aligned}
\frac{1}{2 \tau \sqrt{1 / 4 \tau^{2}-(D / \tau) \tilde{k}_{x}^{2}}}= & \frac{1}{2}, \\
1 & <\frac{1}{2 \tau \sqrt{1 / 4 \tau^{2}-(D / \tau) \tilde{k}_{x}^{2}}}<\infty .
\end{aligned}
$$

Then, solution (67) can be written in its final form:

$$
\begin{aligned}
T(x, t)= & T_{0} \cdot \exp \left(i \widetilde{k}_{x} x\right) \cdot E_{\varphi, 1}\left(-\frac{1}{2} \varphi^{1-\varphi} \widehat{t}^{\varphi}\right) \\
& \cdot E_{\varphi, 1}\left(-\varphi^{1-\varphi} \widehat{t}^{\varphi}\right) .
\end{aligned}
$$

Case 4. When $\varphi=1 / 2$, from (67) we have

$$
\begin{aligned}
T(x, t)= & T_{0} \cdot \exp \left(i \widetilde{k}_{x} x\right) \cdot \exp \left(\frac{\sigma_{t}}{4 \tau^{2}} t\right) \\
& \cdot\left[1-\operatorname{erfc}\left(-\frac{\sigma_{t}^{1 / 2}}{2 \tau} t^{1 / 2}\right)\right] \\
& \cdot \exp \left[\left(\frac{1}{4 \tau^{2}}-\frac{D}{\tau} \widetilde{k}_{x}^{2}\right) \sigma_{t} t\right] \\
& \cdot\left[1-\operatorname{erfc}\left(\frac{1}{4 \tau^{2}}-\frac{D}{\tau} \widetilde{k}_{x}^{2}\right)^{1 / 2} \sigma_{t}^{1 / 2} t^{1 / 2}\right],
\end{aligned}
$$

and $\operatorname{erfc}(\alpha)$ denotes the error function defined in (8). Equation (77) represents the time evolution of the temperature and the amplitude exhibits an algebraic decay for $t \rightarrow \infty$. Plots for different values of $\varphi$ are shown in Figures 3(a) and 3(b). 
Table 3 shows the different solutions of (76). The order of the fractional differential equation is $\varphi=2, \varphi=3 / 2$, $\varphi=1$, and $\varphi=1 / 2$. The change of the order of the derivative describes the crossover from ballistic transport to the diffusion behavior.

3.3. Fractional Space-Time Cattaneo-Vernotte Equation. Now considering (18) and assuming that the space and time derivative are fractional, the order of the time-space fractional differential equations is $\varphi \in(0,1]$; for this example we consider, for $t>0, x=0$ and $x=L$ with Dirichlet condition $T(0, t)=T(L, t)=0$ and initial conditions $0<x<L, t=0$ : $T(t, 0)=T_{0}>0$, and $0<x<L, t=0:\left.(\partial T / \partial t)\right|_{t=0}=0$.

Applying the Fourier method of the variable separation, the full solution of (18) is

$$
\begin{gathered}
T(x, t)=\frac{T_{0}}{\pi \tau} \cdot E_{\varphi}\left(-\frac{1}{2 \tau} \sigma_{t}^{1-\varphi} t^{\varphi}\right) \\
\cdot \sum_{m=1}^{\infty} \frac{1}{2 m-1} \Im\left[E_{i \varphi}\left(\frac{(2 m-1) \pi}{L} \sigma_{x}^{1-\varphi} x^{\varphi}\right)\right] \\
\cdot\left[\frac{-1+2 \tau z_{2 m-1}}{z_{2 m-1}} \cdot E_{\varphi}\left(-z_{2 m-1} \sigma_{t}^{1-\varphi} t^{\varphi}\right)\right. \\
\left.+\frac{1+2 \tau z_{2 m-1}}{z_{2 m-1}} \cdot E_{\varphi}\left(z_{2 m-1} \sigma_{t}^{1-\varphi} t^{\varphi}\right)\right]
\end{gathered}
$$

where $\mathfrak{I}$ indicates the imaginary part and $z_{m}=$ $\sqrt{\left(L^{2}-4 m \pi \tau D\right) / 2 L \tau}$. In the case when $\varphi=1$, we have the classical solution

$$
\begin{aligned}
& T(x, t)=\frac{T_{0}}{\pi \tau} \cdot \exp \left(-\frac{1}{2 \tau} t\right) \cdot \sum_{m=1}^{\infty} \frac{1}{2 m-1} \\
& \cdot \sin \left(\frac{(2 m-1) \pi}{L} x\right) \cdot\left[\frac{-1+2 \tau z_{2 m-1}}{z_{2 m-1}}\right. \\
& \left.\cdot \exp \left(-z_{2 m-1} t\right)+\frac{1+2 \tau z_{2 m-1}}{z_{2 m-1}} \cdot \exp \left(z_{2 m-1} t\right)\right] .
\end{aligned}
$$

Now considering (18) with the Riesz space fractional derivative, the order of the space fractional differential equations is $\varphi \in(0,1]$; for this example we consider, for $0<t \leq T$, $0<x<L$, and Dirichlet condition $T(0, t)=T(L, t)=0$ and initial conditions $T(x, 0)=h(x)$,

$$
\begin{gathered}
\frac{\partial^{2} T(x, t)}{\partial t^{2}}+\frac{1}{\tau} \sigma_{t}^{1-\varphi} \frac{\partial T(x, t)}{\partial t}=-\frac{D}{\tau} \widetilde{k}_{x}^{2} \sigma_{t}^{2(1-\varphi)} \\
\cdot\left[\frac{1}{2 \cos (\pi \varphi / 2)}\right. \\
\cdot\left[\frac{1}{\Gamma(n-\varphi)}\left(\frac{\partial}{\partial x}\right)^{n} \int_{-} \infty^{x} \frac{f(\chi, t) d \chi}{(x-\chi)^{\varphi+1-n}}\right. \\
\left.\left.+\frac{(-1)^{n}}{\Gamma(n-\varphi)}\left(\frac{\partial}{\partial x}\right)^{n} \int_{x}^{\infty} \frac{f(\chi, t) d \chi}{(\chi-x)^{\varphi+1-n}}\right]\right],
\end{gathered}
$$

and the solution is given by

$$
T(x, t)=\sum_{n=1}^{\infty} A_{n}(t) \sin \left(\frac{n \pi x}{L}\right),
$$

which satisfies the boundary condition; substituting this condition into (81) we obtain

$$
\begin{aligned}
\sum_{n=1}^{\infty} & {\left[\frac{d^{2} A_{n}}{d t^{2}}+\frac{1}{\tau} \sigma_{t}^{1-\varphi} \frac{d}{d t} A_{n}\right.} \\
& \left.+\left[\frac{D}{\tau} \widetilde{k}_{x}^{2} \sigma_{t}^{2(1-\varphi)}\left(\lambda_{n}\right)^{\varphi / 2}\right] A_{n}\right] \sin \left(\frac{n \pi x}{L}\right)=0,
\end{aligned}
$$

and the problem for $A_{n}$ becomes

$$
\frac{d^{2} A_{n}}{d t^{2}}+\frac{1}{\tau} \sigma_{t}^{1-\varphi} \frac{d}{d t} A_{n}+\left[\frac{D}{\tau} \widetilde{k}_{x}^{2} \sigma_{t}^{2(1-\varphi)}\left(\lambda_{n}\right)^{\varphi / 2}\right] A_{n}=0
$$

which has the general solution

$$
\begin{aligned}
T(x, t)= & A_{n}(0) \\
& \cdot \exp \left(-\frac{1}{2 \tau}\left(1+\sqrt{1-\frac{4 \tau \widetilde{k}_{x}^{2}}{D}}\right) t\right) \\
& \cdot \exp \left(\frac{D}{\tau} \widetilde{k}_{x}^{2} \sigma_{t}^{2(1-\varphi)}\left(\lambda_{n}\right)^{\varphi / 2} t\right) ;
\end{aligned}
$$

to obtain $A_{n}(0)$, we use the initial condition

$$
T(x, t)=\sum_{n=1}^{\infty} T_{n}(0) \sin \left(\frac{n \pi x}{L}\right)=g(x),
$$

from which we deduce that

$$
T_{n}(0)=\frac{2}{L} \int_{0}^{L}(g \chi) \sin \left(\frac{n \pi \chi}{L}\right) d \chi=B_{n} .
$$

Hence, the solution is given by

$$
\begin{aligned}
& T(x, t) \\
& =\sum_{n=1}^{\infty} B_{n} \sin \left(\frac{n \pi x}{L}\right) \\
& \quad \cdot \exp \left(\left(-\frac{1}{2 \tau} \sigma_{t}^{1-\varphi}-\left(-\frac{1}{4 \tau^{2}}-\frac{D}{\tau} \widetilde{k}_{x}^{2}\right)^{1 / 2} \sigma_{t}^{1-\varphi}\right) t\right) \\
& \quad \cdot \exp \left(-\frac{D}{\tau} \widetilde{k}_{x}^{2} \sigma_{t}^{2(1-\varphi)}\left(\lambda_{n}\right)^{\varphi / 2} t\right),
\end{aligned}
$$

where $\lambda_{n}=n^{2} \pi^{2} / L^{2}$.

\section{Conclusions}

In this paper we introduced an alternative representation of the fractional Cattaneo-Vernotte equation. In particular, 


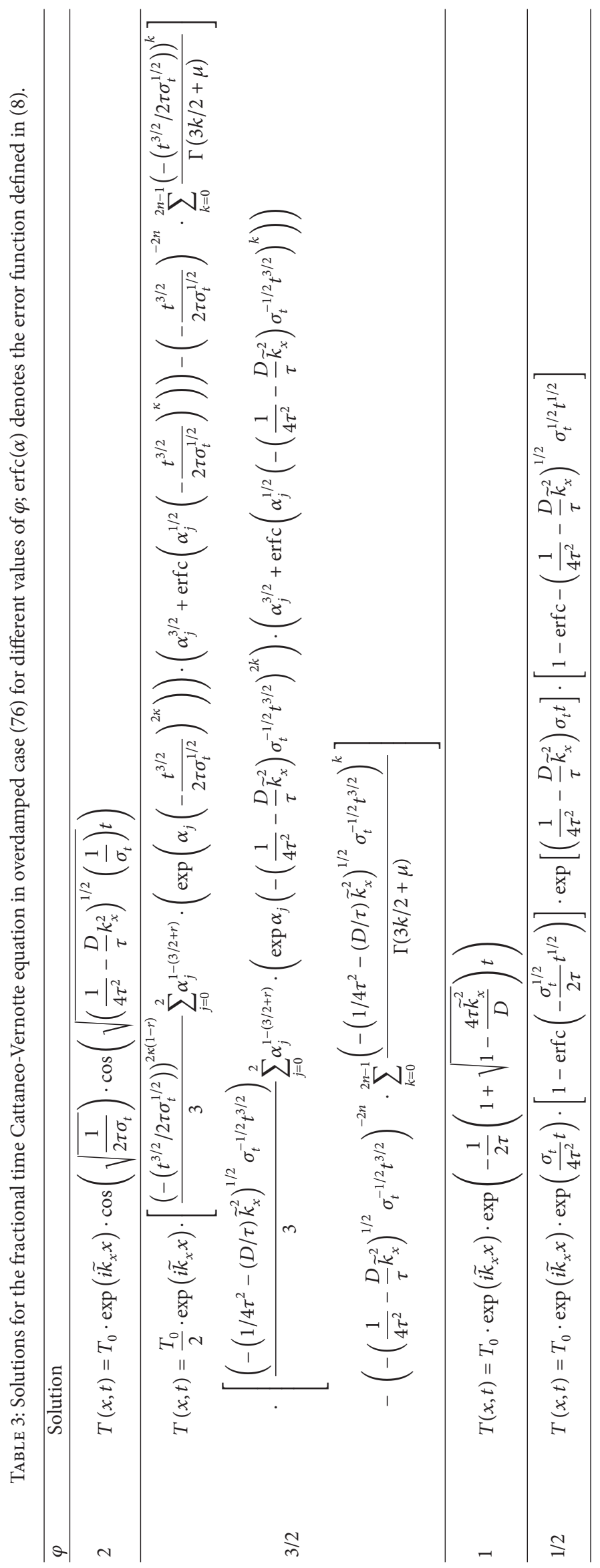


a one-dimensional model was considered in detail. We showed that the fractional Cattaneo-Vernotte equation inherits some crucial characteristics; in particular, the parameters $\sigma_{x}$ and $\sigma_{t}$ need to be introduced in order to characterize the behavior of the physical system, which is located into an intermediate state between a conservative and dissipative system presenting anomalous relaxations. This combination of stored and dissipated energy is conveniently based on the representation of linear thermoviscoelasticity theory. Usually this dissipation is known as internal friction. Some special cases are also discussed. Our results indicate that the fractional order $\varphi$ has an important influence on the temperature. Considering the Dirichlet conditions, in the range $0<\varphi \leq 1$ for the spatial case, we observe the non-Markovian Lévy flights and, in the temporal case for the range $0<\varphi<1$, the subdiffusion phenomena. In the spatial case when $\varphi=3 / 2$ and $\varphi=2$, the diffusion exhibits an increment in the amplitude and the behavior presents anomalous dispersion (the diffusion increases with increasing order of $\varphi$ ), in the range $1<\varphi \leq 2$ we observe the Markovian Lévy flights. Finally, in the temporal case, when $\varphi=3 / 2$ and $\varphi<2$, the diffusion exhibits an increment in the amplitude and presents the superdiffusion, and the case $\varphi=2$ represents the ballistic diffusion. A crossover from a powerlaw behavior for short times to an exponential decay for long times has been found. When memory effects are incorporated using fractional time derivatives, the crossover dynamics is richer. The alternative model and results in this paper provide a new theoretical perspective of the non-Fourier heat conduction. Furthermore, since the solutions are given in terms of the multivariate Mittag-Leffler functions depending only on a small number of parameters, the universality concept (when the class of behavior does not depend on the details of the physical system) can be considered through this methodology since the analytic solutions presented only need a few parameters to describe their behavior; in all cases the solutions preserve the physical units of the system studied.

Among problems for further research we mention the problem of thermal convection of non-Fourier fluids and the non-Newtonian effects in thermal convection (some situations exist where the non-Fourier and non-Newtonian effects are simultaneously present such as rarefied gases with high Knudsen numbers [52-54]; in this case it is important to describe the interaction between the thermal relaxation and viscous (stress) relaxation); another problem is the two- and three-dimensional fractional wave equations considering fractional variational calculus (see [54] and the references therein) with different initial or/and boundary conditions; of course, it would be interesting to consider the fractional thermal wave equations with fractional derivatives defined in different ways. Furthermore, the methodology proposed in this work can be applied in the critical phenomena theory, self-similarity, scale-invariance, propagation of energy in dissipative systems, theory of viscoelastic fluids and solids, relaxing gas dynamics, irreversible thermodynamics, theory of thermal stresses, thermoelasticity, cosmological models, finance modeling, theory of diffusion in crystalline solids, and the description of anomalous complex processes.

\section{Competing Interests}

The authors declare no competing interests.

\section{Acknowledgments}

The authors would like to thank Mayra Martínez for the interesting discussions. J. F. Gómez Aguilar acknowledges the support provided by CONACYT: Cátedras CONACYT para Jovenes Investigadores 2014.

\section{References}

[1] D. D. Joseph and L. Preziosi, "Heat waves," Reviews of Modern Physics, vol. 61, no. 1, pp. 41-73, 1989.

[2] E. K. Lenzi, L. R. Evangelista, M. K. Lenzi, H. V. Ribeiro, and E. C. de Oliveira, "Solutions for a non-Markovian diffusion equation," Physics Letters A, vol. 374, no. 41, pp. 4193-4198, 2010.

[3] D. Ben-Avraham and S. Havlin, Diffusion and Reactions in Fractals and Disordered Systems, Cambridge University Press, Cambridge, UK, 2000.

[4] R. Metzler, A. V. Chechkin, and J. Klafter, "Levy statistics and anomalous transport: levy flights and subdiffusion," in Encyclopedia of Complexity and Systems Science, pp. 5218-5239, 2009.

[5] J. T. Machado, V. Kiryakova, and F. Mainardi, "Recent history of fractional calculus," Communications in Nonlinear Science and Numerical Simulation, vol. 16, no. 3, pp. 1140-1153, 2011.

[6] J. A. Tenreiro Machado, A. M. Galhano, and J. J. Trujillo, "Science metrics on fractional calculus development since 1966," Fractional Calculus and Applied Analysis, vol. 16, no. 2, pp. 479-500, 2013.

[7] J. A. Tenreiro Machado, A. M. S. F. Galhano, and J. J. Trujillo, "On development of fractional calculus during the last fifty years," Scientometrics, vol. 98, no. 1, pp. 577-582, 2014.

[8] T. Sandev, R. Metzler, and Z. Tomovski, "Velocity and displacement correlation functions for fractional generalized Langevin equations," Fractional Calculus and Applied Analysis, vol. 15, no. 3, pp. 426-450, 2012.

[9] C. H. Eab and S. C. Lim, "Fractional Langevin equations of distributed order," Physical Review E, vol. 83, no. 3, Article ID 031136, 10 pages, 2011.

[10] P. De Anna, T. Le Borgne, M. Dentz, A. M. Tartakovsky, D. Bolster, and P. Davy, "Flow intermittency, dispersion, and correlated continuous time random walks in porous media," Physical Review Letters, vol. 110, no. 18, Article ID 184502, 2013.

[11] M. Magdziarz, R. Metzler, W. Szczotka, and P. Zebrowski, "Correlated continuous-time random walks in external force fields," Physical Review E-Statistical, Nonlinear, and Soft Matter Physics, vol. 85, no. 5, Article ID 051103, 2012.

[12] A. Zeb, G. Zaman, I. H. Jung, and M. Khan, "Optimal campaign strategies in fractional-order smoking dynamics," Zeitschrift für Naturforschung A, vol. 69, no. 5-6, pp. 225-231, 2014.

[13] I. S. Jesus and J. A. Tenreiro Machado, "Fractional control of heat diffusion systems," Nonlinear Dynamics, vol. 54, no. 3, pp. 263-282, 2008.

[14] M. Zingales, "Fractional-order theory of heat transport in rigid bodies," Communications in Nonlinear Science and Numerical Simulation, vol. 19, no. 11, pp. 3938-3953, 2014. 
[15] J. F. Gómez-Aguilar and D. Baleanu, "Fractional transmission line with losses," Zeitschrift fur Naturforschung, vol. 69, no. 1011, pp. 539-546, 2014.

[16] J. F. Gómez Aguilar and D. Baleanu, "Solutions of the telegraph equations using a fractional calculus approach," Proceedings of the Romanian Academy: Series A, vol. 15, no. 1, pp. 27-34, 2014.

[17] V. Mishra, K. Vishal, S. Das, and S. H. Ong, "On the solution of the nonlinear fractional diffusion-wave equation with absorption: a homotopy approach," Zeitschrift für Naturforschung A, vol. 69, no. 3-4, pp. 135-144, 2014.

[18] R. T. Sibatov and V. V. Uchaikin, "Fractional differential approach to dispersive transport in semiconductors," PhysicsUspekhi, vol. 52, no. 10, pp. 1019-1043, 2009.

[19] X.-J. Yang, D. Baleanu, and H. M. Srivastava, "Local fractional similarity solution for the diffusion equation defined on Cantor sets," Applied Mathematics Letters, vol. 47, pp. 54-60, 2015.

[20] R. Metzler and J. Klafter, “The random walk's guide to anomalous diffusion: a fractional dynamics approach," Physics Reports, vol. 339, no. 1, pp. 1-77, 2000.

[21] G.-C. Wu, D. Baleanu, S.-D. Zeng, and Z.-G. Deng, "Discrete fractional diffusion equation," Nonlinear Dynamics, vol. 80, no. 1-2, pp. 281-286, 2015.

[22] O. Defterli, M. D’Elia, Q. Du, M. Gunzburger, R. Lehoucq, and M. M. Meerschaert, "Fractional diffusion on bounded domains," Fractional Calculus and Applied Analysis, vol. 18, no. 2, pp. 342-360, 2015.

[23] R. Schumer, M. M. Meerschaert, and B. Baeumer, "Fractional advection-dispersion equations for modeling transport at the earth surface," Journal of Geophysical Research: Earth Surface, vol. 114, no. 4, Article ID F00A07, 2009.

[24] M. M. Meerschaert, D. A. Benson, H.-P. Scheffler, and B. Baeumer, "Stochastic solution of space-time fractional diffusion equations," Physical Review E, vol. 65, no. 4, Article ID 041103, 4 pages, 2002.

[25] F. Liu, M. M. Meerschaert, R. J. McGough, P. Zhuang, and Q. Liu, "Numerical methods for solving the multi-term timefractional wave-diffusion equation," Fractional Calculus and Applied Analysis, vol. 16, no. 1, pp. 9-25, 2013.

[26] A. Kullberg, D. del-Castillo-Negrete, G. J. Morales, and J. E. Maggs, "Isotropic model of fractional transport in twodimensional bounded domains," Physical Review E, vol. 87, no. 5, Article ID 052115, 2013.

[27] R. Gorenflo, Y. Luchko, and M. Stojanovi, "Fundamental solution of a distributed order time-fractional diffusion-wave equation as probability density," Fractional Calculus and Applied Analysis, vol. 16, no. 2, pp. 297-316, 2013.

[28] F. Mainardi, Y. Luchko, and G. Pagnini, “The fundamental solution of the space-time fractional diffusion equation," Fractional Calculus and Applied Analysis, vol. 4, no. 2, pp. 153-192, 2001.

[29] J. F. G. Aguilar and M. M. Hernández, "Space-time fractional diffusion-advection equation with Caputo derivative," Abstract and Applied Analysis, vol. 2014, Article ID 283019, 8 pages, 2014.

[30] R. Gorenflo, F. Mainardi, D. Moretti, and P. Paradisi, “Time fractional diffusion: a discrete random walk approach," Nonlinear Dynamics, vol. 29, no. 1, pp. 129-143, 2002.

[31] A. Compte and R. Metzler, "The generalized cattaneo equation for the description of anomalous transport processes," Journal of Physics A: Mathematical and General, vol. 30, no. 21, pp. 72777289, 1997.

[32] Y. Postvenko, "Theories of termal stresses based on space-timefractional telegraph equations," Computers and Mathematics with Applications, vol. 64, no. 10, pp. 3321-3328, 2012.
[33] T. Atanackovic, S. Konjik, L. Oparnica, and D. Zorica, "The Cattaneo type space-time fractional heat conduction equation," Continuum Mechanics and Thermodynamics, vol. 24, no. 4-6, pp. 293-311, 2012.

[34] T. M. Atanackovic, S. Pilipovic, and D. Zorica, "A diffusion wave equation with two fractional derivatives of different order," Journal of Physics A: Mathematical and Theoretical, vol. 40, no. 20, pp. 5319-5333, 2007.

[35] K. D. Lewandowska and T. Kosztołowicz, "Application of generalized cattaneo equation to model subdiffusion impedance," Acta Physica Polonica B, vol. 39, no. 5, pp. 1211-1220, 2008.

[36] V. E. Tarasov, "Transport equations from Liouville equations for fractional systems," International Journal of Modern Physics B. Condensed Matter Physics. Statistical Physics. Applied Physics, vol. 20, no. 3, pp. 341-353, 2006.

[37] H. T. Qi and X. Y. Jiang, "Solutions of the space-time fractional Cattaneo diffusion equation," Physica A, vol. 390, no. 11, pp. 1876-1883, 2011.

[38] H.-Y. Xu, H.-T. Qi, and X.-Y. Jiang, "Fractional Cattaneo heat equation in a semi-infinite medium," Chinese Physics B, vol. 22, no. 1, Article ID 014401, 6 pages, 2013.

[39] Y. J. Yu, X. G. Tian, and T. J. Lu, "Fractional order generalized electro- magneto-thermo-elasticity," European Journal of Mechanics-A/Solids, vol. 42, pp. 188-202, 2013.

[40] S.-W. Vong, H.-K. Pang, and X.-Q. Jin, "A high-order difference scheme for the generalized Cattaneo equation," East Asian Journal on Applied Mathematics, vol. 2, no. 2, pp. 170-184, 2012.

[41] X. Zhao and Z.-Z. Sun, "Compact Crank-Nicolson schemes for a class of fractional Cattaneo equation in inhomogeneous medium," Journal of Scientific Computing, vol. 62, no. 3, pp. 747771, 2015.

[42] J. F. Gómez-Aguilar, M. Miranda-Hernández, M. G. LópezLópez, V. M. Alvarado-Martínez, and D. Baleanu, "Modeling and simulation of the fractional space-time diffusion equation," Communications in Nonlinear Science and Numerical Simulation, vol. 30, no. 1-3, pp. 115-127, 2016.

[43] K. S. Miller and B. Ross, An Introduction to the Fractional Calculus and Fractional Differential Equations, John Wiley \& Sons, New York, NY, USA, 1993.

[44] I. Podlubny, Fractional Differential Equations, Academic Press, New York, NY, USA, 1999.

[45] D. Baleanu, K. Diethelm, E. Scalas, and J. J. Trujillo, Fractional Calculus Models and Numerical Methods, Complexity, Nonlinearity and Chaos, World Scientific, 2012.

[46] K. Diethelm, N. J. Ford, A. D. Freed, and Y. Luchko, "Algorithms for the fractional calculus: a selection of numerical methods," Computer Methods in Applied Mechanics and Engineering, vol. 194, no. 6-8, pp. 743-773, 2005.

[47] K. Diethelm, The Analysis of Fractional Differential Equations. An Application-Oriented Exposition Using Differential Operators of Caputo Type, Springer, Berlin, Germany, 2010.

[48] H. J. Haubold, A. M. Mathai, and R. K. Saxena, "MittagLeffler functions and their applications," Journal of Applied Mathematics, vol. 2011, Article ID 298628, 51 pages, 2011.

[49] K. S. Miller, "Some simple representations of the generalized Mittag-Leffler functions," Integral Transforms and Special Functions, vol. 11, no. 1, pp. 13-24, 2001.

[50] R. Garrappa and M. Popolizio, "Evaluation of generalized Mittag-Leffler functions on the real line," Advances in Computational Mathematics, vol. 39, no. 1, pp. 205-225, 2013. 
[51] H. Struchtrup and P. Taheri, "Macroscopic transport models for rarefied gas flows: a brief review," IMA Journal of Applied Mathematics, vol. 76, no. 5, pp. 672-697, 2011.

[52] H. Brenner, "Steady-state heat conduction in a gas undergoing rigid-body rotation. Comparison of Navier-Stokes-Fourier and bivelocity paradigms," International Journal of Engineering Science, vol. 70, pp. 29-45, 2013.

[53] H. Struchtrup, "Resonance in rarefied gases," Continuum Mechanics and Thermodynamics, vol. 24, no. 4-6, pp. 361-376, 2012.

[54] V. E. Tarasov, "Fractional vector calculus and fractional Maxwell's equations," Annals of Physics, vol. 323, no. 11, pp. 2756-2778, 2008. 


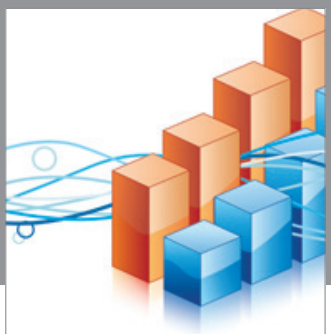

Advances in

Operations Research

vatem alat4

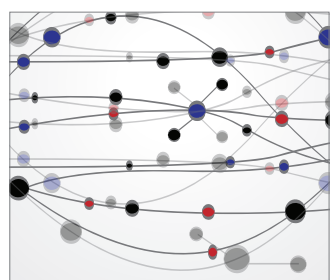

\section{The Scientific} World Journal
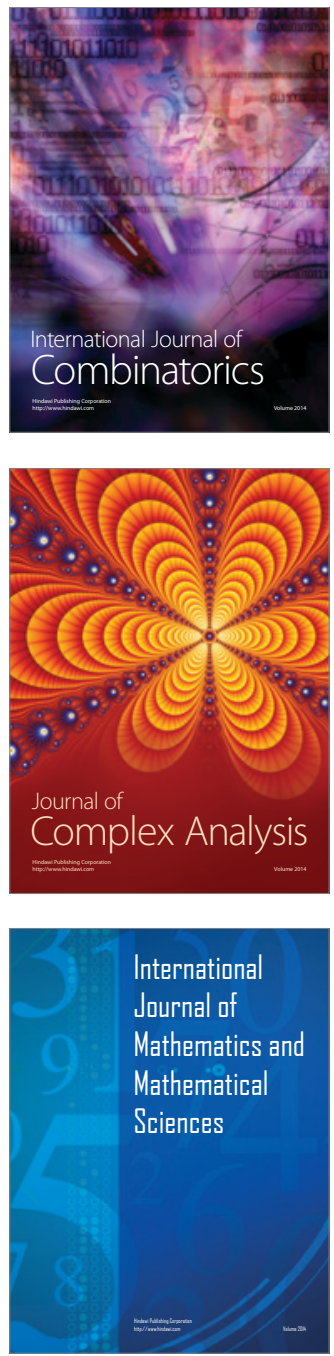
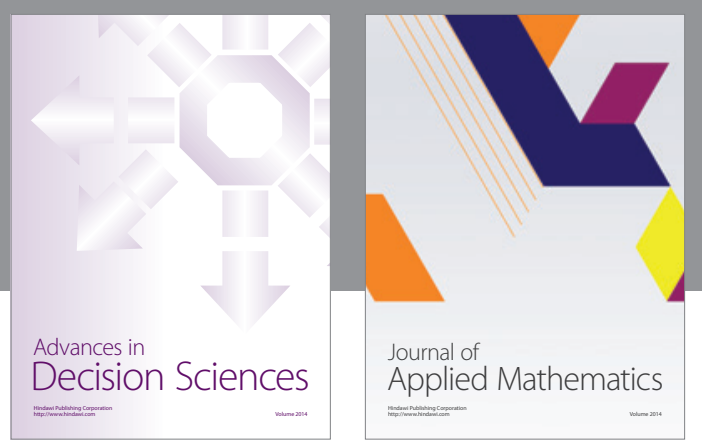

Algebra

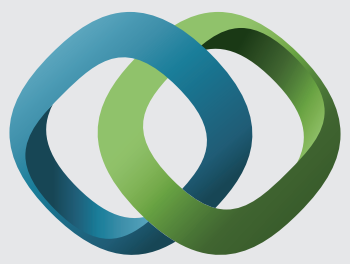

\section{Hindawi}

Submit your manuscripts at

http://www.hindawi.com
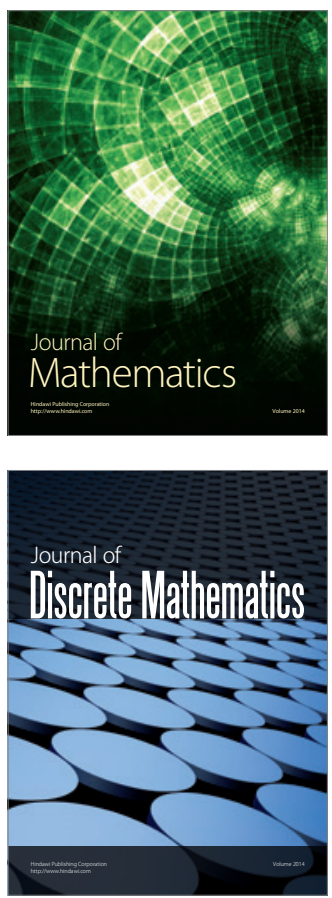

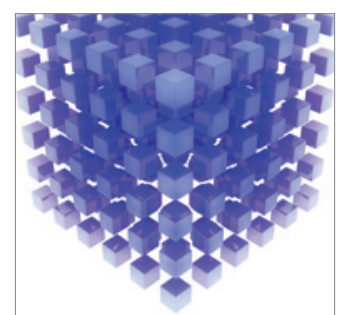

Mathematical Problems in Engineering
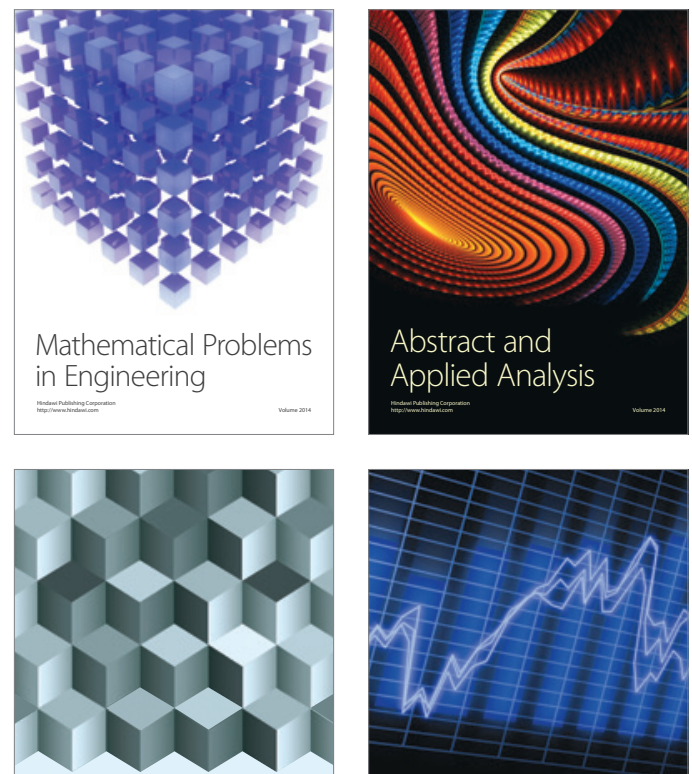

Journal of

Function Spaces

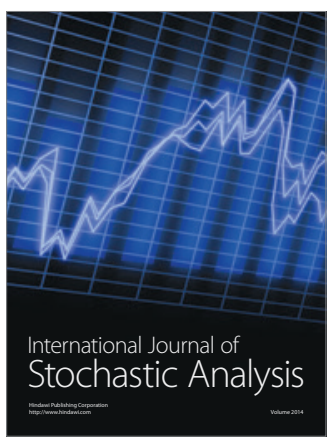

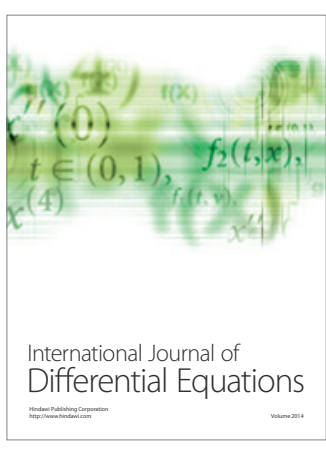
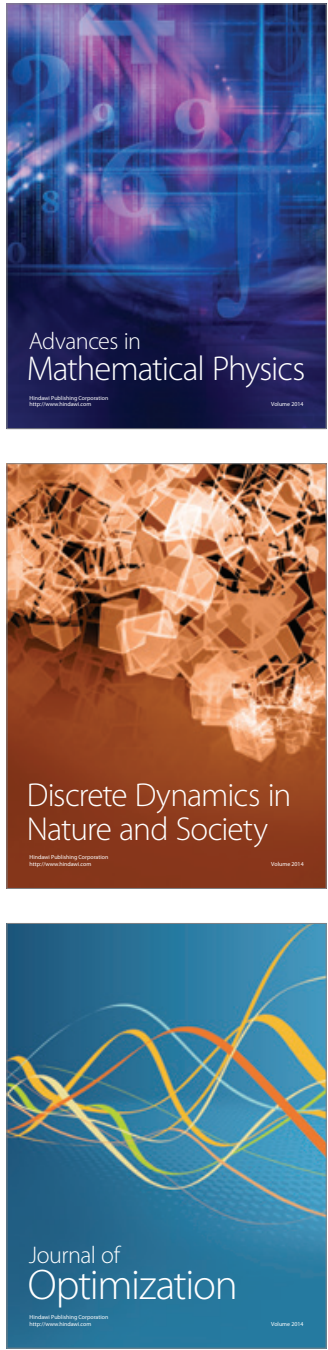